\title{
Integrative analysis of multi-omics data for identifying multi-markers for diagnosing pancreatic cancer
}

\author{
Min-Seok Kwon', Yongkang Kim², Seungyeoun Lee ${ }^{3}$, Junghyun Namkung ${ }^{4}$, Taegyun Yun ${ }^{4}$, Sung Gon Yi ${ }^{4}$, \\ Sangjo Han ${ }^{4}$, Meejoo Kang ${ }^{5}$, Sun Whe Kim ${ }^{5}$, Jin-Young Jang ${ }^{5^{*}}$, Taesung Park ${ }^{1,2^{*}}$
}

From IEEE International Conference on Bioinformatics and Biomedicine (BIBM 2014)

Belfast, UK. 2-5 November 2014

\begin{abstract}
Background: microRNA (miRNA) expression plays an influential role in cancer classification and malignancy, and miRNAs are feasible as alternative diagnostic markers for pancreatic cancer, a highly aggressive neoplasm with silent early symptoms, high metastatic potential, and resistance to conventional therapies.

Methods: In this study, we evaluated the benefits of multi-omics data analysis by integrating miRNA and mRNA expression data in pancreatic cancer. Using support vector machine (SVM) modelling and leave-one-out cross validation (LOOCV), we evaluated the diagnostic performance of single- or multi-markers based on miRNA and mRNA expression profiles from 104 PDAC tissues and 17 benign pancreatic tissues. For selecting even more reliable and robust markers, we performed validation by independent datasets from the Gene Expression Omnibus (GEO) and the Cancer Genome Atlas (TCGA) data depositories. For validation, miRNA activity was estimated by miRNA-target gene interaction and mRNA expression datasets in pancreatic cancer.

Results: Using a comprehensive identification approach, we successfully identified 705 multi-markers having powerful diagnostic performance for PDAC. In addition, these marker candidates annotated with cancer pathways using gene ontology analysis.

Conclusions: Our prediction models have strong potential for the diagnosis of pancreatic cancer.
\end{abstract}

\section{Background}

The development of early diagnostic biomarkers and innovative therapeutic strategies to prevent the progression of cancers is urgent. However, common biomarker development strategies, based on gene expression alone, have only limited potential to identify novel biomarkers. Due several distinguishing characteristics, microRNAs (miRNAs) have become new potential biomarkers in cancer genetics. miRNAs are small noncoding RNA molecules which "micro-manage" messenger RNA (mRNA) expression by reducing its translation and

\footnotetext{
*Correspondence: jangjy4@gmail.com; tspark@stats.snu.ac.kr

'Interdisciplinary program in Bioinformatics, Seoul National University, Seoul, Korea

${ }^{5}$ Department of Surgery, Seoul National University Hospital, Seoul, Korea
} Full list of author information is available at the end of the article stability [1]. Recent studies show that in particular, miRNAs play a crucial role in cancer cell proliferation [2], apoptosis [3], angiogenesis [4], metastasis [5], and chemoresistance [6] by changing the expression of both oncogenes and tumor suppressors [7] in pancreatic cancer. These biological roles of miRNAs represent their potential as diagnostic biomarkers for pancreatic cancer.

An important step of estimating the gene-regulatory activity of miRNAs is accurately predicting their targets and monitoring their expression levels. Several computational target prediction tools have been developed, such as TargetScan version 6.2 [8], PITA version hg18 [9], and miRvestigator [10]. However, these in silico target prediction tools suffer from high false positive rates because the tools use only sequence complementarity 
and assume structural stability (following putative assembly) to predict a specific miRNA's target [11]. As miRNA regulatory activation often depends on the distinct tissue being studied (e.g., cancer tissue), the use of condition (i.e., stress, S-phase, etc.)-specific miRNA and mRNA expression data is required to find true miRNA activity [12]. Therefore, the use of miRNAs as potential biomarkers in dismal cancers such as pancreatic cancer remains difficult.

Pancreatic cancer is one of the most hard-to-diagnose and aggressive malignancies, despite increasing knowledge of its etiology [13]. Because of its highly lethal nature and silent symptoms, pancreatic cancer has remained one of the leading causes of cancer-related death [14]. Among the several types of pancreatic cancers, pancreatic ductal adenocarcinoma (PDAC) is the most abundant cancer type which accounts for about $85 \%$ of exocrine pancreatic cancers. Although recent advances in gene expression profiling technology, such as microarray and massively parallel sequencing, enable researchers to discover gene-based biomarkers for PDAC diagnosis, there are no highly effective diagnostic markers for PDAC. In order to improve the survival rate of PDAC patients, it is important to identify efficient diagnostic, prognostic, and therapy response markers.

In this study, we performed a novel approach to identify diagnostic markers for PDAC by integrating
miRNA and mRNA expression profiles. Using paired miRNA and mRNA expression profiling, we successfully identified promising mRNA and miRNA markers. By determining differential miRNA expression profiles and interaction with their target genes in PDAC, as compared to normal pancreatic tissues, we estimated miRNA expression levels in independent datasets lacking miRNA expression (i.e., having mRNA data only), and validated the diagnostic performance of miRNA marker candidates.

\section{Results and discussion}

In this section, we firstly identified multi-markers using mRNA and miRNA expression data from 104 PDAC tissues and 17 benign pancreatic tissues, using support vector machine (SVM) classification and leave-one-out cross-validation (LOOCV). Then, using miRNA target interactions constructed using publically available target prediction tools, we validated marker candidates in independent datasets to select more reliable markers. In the case of independent datasets lacking miRNA expression, we used estimated miRNA activity for validation (based on the expression levels of the miRNA target mRNA transcripts). After validation of the selected candidates, we used other cancer datasets to evaluate and annotate their functions, as shown in Figures 1 and 2 .

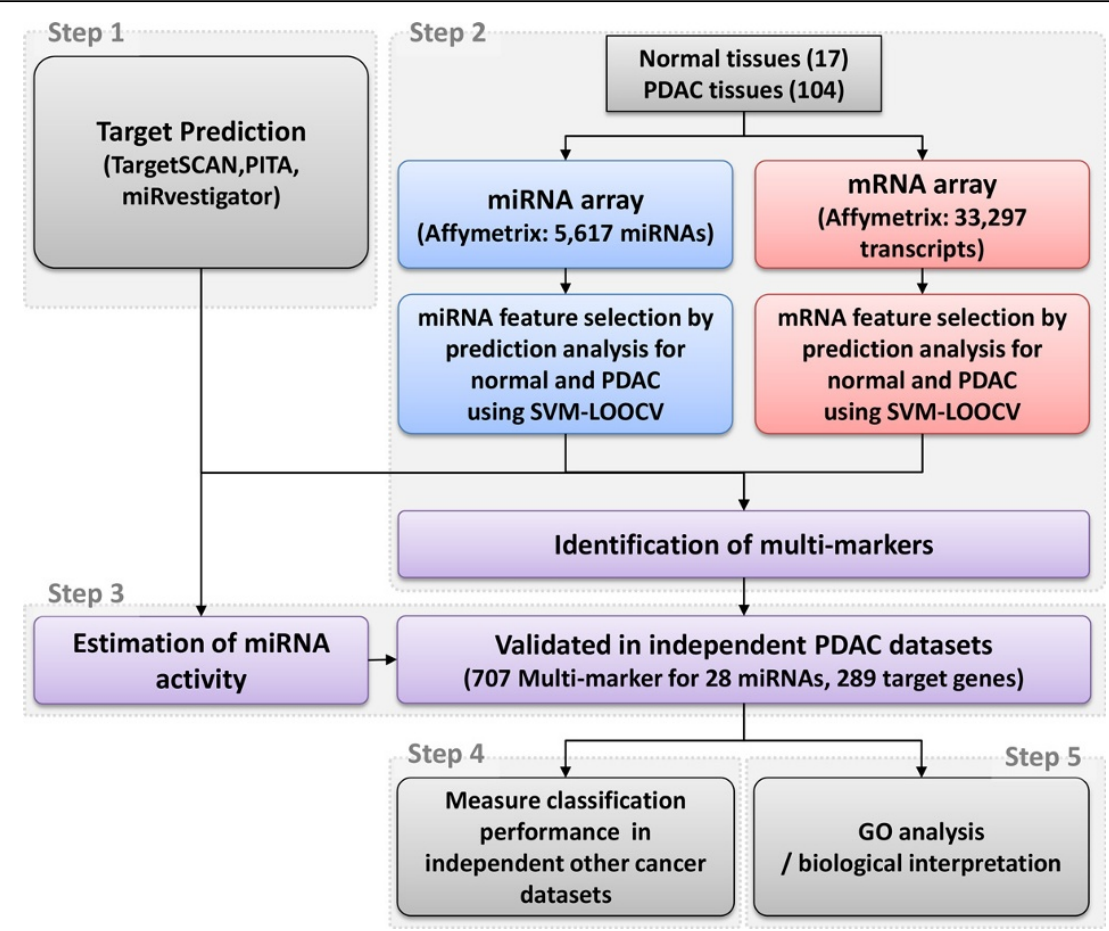

Figure 1 An analysis scheme of our integrated analysis for PDAC. 104 PDAC tumor and 17 normal pancreatic tissues were separately analysed for gene and miRNA expression using microarrays. Specific features of miRNAs and mRNAs were modelled by SVM and leave-one-out cross-validation (LOOCV). These were then verified by miRNA target prediction algorithms and finally, validated in independent datasets. 


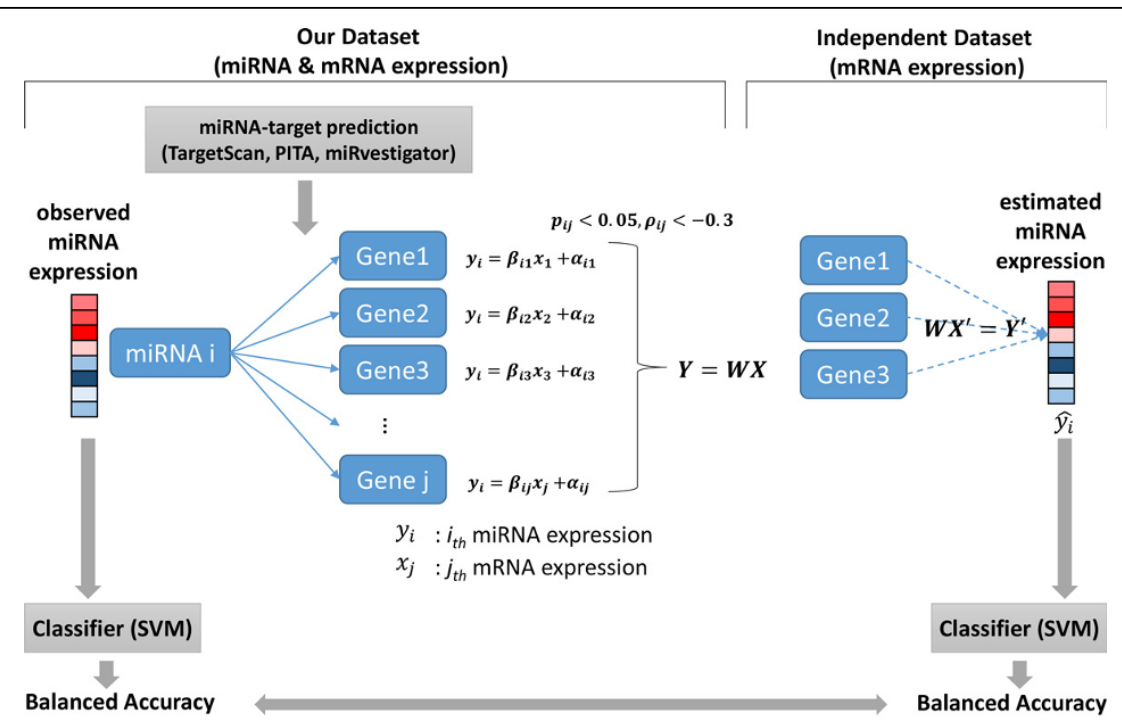

Figure 2 Estimation scheme miRNA expression. Based on the predicted targeting activity of specific miRNAs and their targets identified by three miRNA target prediction algorithms, we used linear regression to determine mRNA levels and balanced accuracies for both miRNAs and their specific target transcript mRNAs.

\section{Identification of multi-marker candidates from PDAC expression data}

For identification of multi-marker candidates for PDAC, we used miRNA and mRNA expression data from 121 total pancreatic tissues of 104 PDAC tumors and 17 benign tissues [15]. To prevent overfitting of imbalanced data, LOOCV and SVM with sample class weights were applied, as described in the Methods section. After evaluation analysis using PDAC and independent datasets, we identified 705 multi-markers for 27 miRNAs, and 289 genes for PDAC diagnosis.

Table 1 shows the 39 identified multi-markers with high accuracy (BAs > 0.85 and $A U C>0.85$ in our dataset) for diagnosis of PDAC in our training datasets and independent datasets. Specifically, miR-107 was upregulated in PDAC, and miR-107 was recently found to be silenced by promoter DNA methylation in pancreatic cancer [16]. However, DNA demethylation events could induce miR-107 expression showing that epigenetic mechanisms regulating miRNA levels may be involved in pancreatic carcinogenesis. Likewise, miR-135b was reported as a biomarker for PDAC [17], ovarian cancer, and colon cancer [18], in which it promotes proliferation, invasion, and metastasis [19], and miR-135b was similarly upregulated in our findings. By contrast, downregulation of miR-148a was reported in pancreatic, bladder, and lung cancers, and miR-148a was preventative of tumor angiogenesis and cancer progression [20]. miR-21 is also a wellknown potential biomarker for diagnosis, prognosis, and chemosensitivity of pancreatic cancer. As most miR-21 targets are tumor suppressors, miR-21 is associated with various cancers such as those of the breast, ovary, cervix, colon, lung, liver, brain, esophagus, prostate, pancreas, and thyroid [21]. miR-222 has also been reported as differentially expressed in most pancreatic cancers, in which it promotes poor survival rates [22].

In Table 2, 27 miRNAs were identified for efficacy in the diagnosis of PDAC. Of these, 22 were previously known to be differentially expressed in pancreatic cancer [7]. However, miR-941, miR-28, mir-487a, mir-299, and mir-503 have never been reported in pancreatic cancer.

Out of 289 target genes, 142 were coregulated by more than one miRNA. Table 3 lists 17 target genes that were coregulated by more than 6 miRNAs. Although there are complex interactions between these target genes and miRNAs, their expression direction was required to be negatively correlated (e.g., miRNAs upregulated and targets downregulated) for PDAC vs. normal conditions in miRNA-target gene network (Figure 3). The function of most co-regulated target genes correlated with cancer metabolism and cancer progression, through such processes as attenuated apoptosis, abnormal development, angiogenesis, and transcriptional dysregulation.

\section{Estimating the relationship between miRNA activity and miRNA targets}

In our previous study [15], we used the average balanced accuracy (BA), i.e., the arithmetic mean of sensitivity and specificity of target-genes, as a metric for miRNA activity performance. In this paper, we modified the estimation algorithm to improve accuracy of miRNA activity (Figure 2). The main difference was that reliable miRNAtarget gene relationships were determined by testing pancreatic cancer datasets for estimating miRNA activity. 
Table 1 Performance of multi-markers.

\begin{tabular}{|c|c|c|c|c|c|c|c|c|c|c|c|c|c|c|}
\hline \multicolumn{7}{|c|}{ miRNA } & \multicolumn{8}{|c|}{ Target gene } \\
\hline & \multicolumn{3}{|c|}{ PDAC dataset } & \multicolumn{3}{|c|}{ Independent dataset } & \multirow[b]{2}{*}{ target gene } & \multicolumn{4}{|c|}{ PDAC dataset } & \multicolumn{3}{|c|}{ Independent dataset } \\
\hline miRNA & regulation & $B A$ & AUC & PDAC1 & PDAC2 & PDAC3 & & corr $^{a}$ & $p$-value ${ }^{b}$ & $B A$ & AUC & PDAC1 & PDAC2 & PDAC3 \\
\hline \multirow[t]{10}{*}{ miR-107 } & up & 0.859 & 0.851 & 0.800 & 0.729 & 0.670 & DTNA & -0.625 & $1.34 \mathrm{E}-14$ & 0.936 & 0.937 & 0.937 & 0.795 & 0.810 \\
\hline & & & & & & & IFRD1 & -0.593 & $6.44 \mathrm{E}-13$ & 0.932 & 0.988 & 0.949 & 0.782 & 0.550 \\
\hline & & & & & & & KIAA1324 & -0.636 & $3.30 \mathrm{E}-15$ & 0.932 & 0.975 & 0.920 & 0.795 & 0.762 \\
\hline & & & & & & & BTG2 & -0.629 & $8.12 \mathrm{E}-15$ & 0.917 & 0.982 & 0.800 & 0.705 & 0.550 \\
\hline & & & & & & & NTRK2 & -0.499 & 4.83E-09 & 0.889 & 0.905 & 0.823 & 0.705 & 0.772 \\
\hline & & & & & & & VTCN1 & -0.309 & 5.39E-04 & 0.880 & 0.748 & 0.829 & 0.705 & 0.720 \\
\hline & & & & & & & SGK1 & -0.451 & $1.85 \mathrm{E}-07$ & 0.871 & 0.852 & 0.817 & 0.667 & 0.550 \\
\hline & & & & & & & ATP8A 1 & -0.427 & $9.36 \mathrm{E}-07$ & 0.864 & 0.882 & 1.000 & 0.769 & 0.678 \\
\hline & & & & & & & USP2 & -0.464 & 7.14E-08 & 0.864 & 0.894 & 0.960 & 0.744 & 0.633 \\
\hline & & & & & & & PHF17 & -0.600 & $2.80 \mathrm{E}-13$ & 0.863 & 0.941 & 0.954 & 0.705 & 0.932 \\
\hline \multirow[t]{10}{*}{ miR-135b } & up & 0.870 & 0.935 & 0.869 & 0.708 & 0.713 & BACE1 & -0.599 & $3.18 \mathrm{E}-13$ & 0.941 & 0.967 & 1.000 & 0.821 & 0.786 \\
\hline & & & & & & & DTNA & -0.525 & $5.24 \mathrm{E}-10$ & 0.936 & 0.937 & 1.000 & 0.795 & 0.810 \\
\hline & & & & & & & PELI2 & -0.528 & $4.08 \mathrm{E}-10$ & 0.927 & 0.973 & 1.000 & 0.769 & 0.772 \\
\hline & & & & & & & VLDLR & -0.635 & 4.25E-15 & 0.922 & 0.969 & 1.000 & 0.756 & 0.741 \\
\hline & & & & & & & RRBP1 & -0.388 & 1.03E-05 & 0.913 & 0.995 & 1.000 & 0.821 & 0.550 \\
\hline & & & & & & & MKNK1 & -0.603 & $1.88 \mathrm{E}-13$ & 0.902 & 0.953 & 1.000 & 0.744 & 0.786 \\
\hline & & & & & & & BCAT1 & -0.524 & $6.04 \mathrm{E}-10$ & 0.893 & 0.939 & 1.000 & 0.859 & 0.713 \\
\hline & & & & & & & SEMA6D & -0.498 & $5.38 \mathrm{E}-09$ & 0.893 & 0.904 & 1.000 & 0.769 & 0.762 \\
\hline & & & & & & & ATP8A1 & -0.437 & 4.95E-07 & 0.864 & 0.882 & 1.000 & 0.769 & 0.678 \\
\hline & & & & & & & PHF17 & -0.575 & 4.54E-12 & 0.863 & 0.941 & 1.000 & 0.705 & 0.932 \\
\hline \multirow[t]{7}{*}{ miR-148a } & down & 0.927 & 0.956 & 0.897 & 0.788 & 0.688 & SLC2A1 & -0.486 & $1.41 \mathrm{E}-08$ & 0.962 & 0.987 & 0.914 & 0.756 & 0.550 \\
\hline & & & & & & & МВОАТ2 & -0.404 & $3.96 \mathrm{E}-06$ & 0.929 & 0.951 & 0.926 & 0.872 & 0.869 \\
\hline & & & & & & & TRAK1 & -0.371 & 2.60E-05 & 0.905 & 0.973 & 0.863 & 0.692 & 0.793 \\
\hline & & & & & & & SULF1 & -0.494 & 7.54E-09 & 0.878 & 0.864 & 0.800 & 0.923 & 0.755 \\
\hline & & & & & & & KLF5 & -0.425 & 1.10E-06 & 0.870 & 0.870 & 0.926 & 0.769 & 0.835 \\
\hline & & & & & & & LRCH1 & -0.312 & 4.63E-04 & 0.865 & 0.916 & 0.909 & 0.654 & 0.772 \\
\hline & & & & & & & ETV1 & -0.325 & $2.57 \mathrm{E}-04$ & 0.855 & 0.875 & 1.000 & 0.846 & 0.724 \\
\hline \multirow[t]{9}{*}{ miR-21 } & up & 0.897 & 0.925 & 0.903 & 0.725 & 0.687 & DTNA & -0.559 & $2.28 \mathrm{E}-11$ & 0.936 & 0.937 & 0.937 & 0.795 & 0.810 \\
\hline & & & & & & & IFRD1 & -0.532 & $2.80 \mathrm{E}-10$ & 0.932 & 0.988 & 0.949 & 0.782 & 0.550 \\
\hline & & & & & & & BTG2 & -0.648 & $6.89 \mathrm{E}-16$ & 0.917 & 0.982 & 0.800 & 0.705 & 0.550 \\
\hline & & & & & & & BCAT1 & -0.551 & $5.04 \mathrm{E}-11$ & 0.893 & 0.939 & 0.903 & 0.859 & 0.713 \\
\hline & & & & & & & NTRK2 & -0.444 & $2.92 \mathrm{E}-07$ & 0.889 & 0.905 & 0.823 & 0.692 & 0.772 \\
\hline & & & & & & & LIFR & -0.596 & 4.64E-13 & 0.888 & 0.964 & 0.903 & 0.769 & 0.918 \\
\hline & & & & & & & ACAT1 & -0.511 & 1.81 E-09 & 0.875 & 0.830 & 1.000 & 0.795 & 0.550 \\
\hline & & & & & & & PHF17 & -0.609 & $1.03 \mathrm{E}-13$ & 0.863 & 0.941 & 0.954 & 0.705 & 0.932 \\
\hline & & & & & & & SNTB1 & -0.449 & 2.21E-07 & 0.855 & 0.802 & 1.000 & 0.769 & 0.585 \\
\hline miR-222 & up & 0.924 & 1.012 & 0.869 & 0.736 & 0.759 & CXCL12 & -0.452 & 1.69E-07 & 0.932 & 0.970 & 0.851 & 0.705 & 0.932 \\
\hline \multirow[t]{2}{*}{ miR-34a } & up & 0.908 & 0.912 & 0.806 & 0.742 & 0.670 & DTNA & -0.447 & 2.43E-07 & 0.936 & 0.937 & 0.937 & 0.795 & 0.810 \\
\hline & & & & & & & BCAT1 & -0.514 & 1.46E-09 & 0.893 & 0.939 & 0.903 & 0.859 & 0.713 \\
\hline
\end{tabular}

a.correlation coefficient between miRNA mRNA expression. ${ }^{\mathrm{b}} \mathrm{p}$-value from linear regression with miRNA and mRNA expression.

Using GSE32688 dataset [23] with both mRNA expression and miRNA expression, we evaluated our current and previous miRNA estimation algorithm by comparing the estimated and observed BAs of specific miRNAs. The mean-squared errors were 0.01515 and 0.04877 for our new and previous miRNA estimation algorithms, respectively.

\section{Diagnostic performance of selected markers in other} cancers

Using our selected PDAC multi-markers, we evaluated their diagnostic performance in lymphoma and breast, hepatocellular, and lung cancers. All independent datasets were collected from the GEO. Figure 4 presents our selected multi-markers for the four other cancers. Most 
Table 2 Performances of selected 27 miRNAs.

\begin{tabular}{|c|c|c|c|c|c|c|c|}
\hline \multirow[b]{2}{*}{ miRNA } & \multicolumn{4}{|c|}{ PDAC dataset } & \multicolumn{3}{|c|}{ Independent PDAC dataset } \\
\hline & regulation & \# target genes & BA & AUC & PDAC1 & PDAC2 & PDAC3 \\
\hline miR-148a & down & 18 & 0.927 & 0.956 & 0.897 & 0.788 & 0.688 \\
\hline miR-222 & up & 4 & 0.924 & 0.962 & 0.869 & 0.736 & 0.759 \\
\hline miR-100 & up & 11 & 0.923 & 0.957 & 0.794 & 0.734 & 0.656 \\
\hline miR-216b & down & 4 & 0.922 & 0.972 & 0.777 & 0.748 & 0.702 \\
\hline miR-155 & up & 24 & 0.912 & 0.949 & 0.726 & 0.740 & 0.635 \\
\hline miR-203 & up & 74 & 0.899 & 0.921 & 0.703 & 0.717 & 0.676 \\
\hline miR-23a & up & 136 & 0.898 & 0.987 & 0.703 & 0.726 & 0.685 \\
\hline $\operatorname{miR}-21$ & up & 33 & 0.897 & 0.925 & 0.903 & 0.725 & 0.687 \\
\hline miR-130b & down & 20 & 0.897 & 0.981 & 0.771 & 0.762 & 0.654 \\
\hline miR-196b & up & 1 & 0.890 & 0.868 & 0.789 & 0.738 & 0.669 \\
\hline let-7i & up & 29 & 0.883 & 0.948 & 0.720 & 0.746 & 0.681 \\
\hline miR-1825 & down & 8 & 0.881 & 0.833 & 0.760 & 0.745 & 0.633 \\
\hline miR-135b & up & 13 & 0.870 & 0.935 & 0.869 & 0.708 & 0.713 \\
\hline miR-941 & up & 1 & 0.864 & 0.849 & 0.749 & 0.760 & 0.553 \\
\hline miR-28 & up & 20 & 0.860 & 0.898 & 0.749 & 0.744 & 0.685 \\
\hline miR-107 & up & 40 & 0.859 & 0.851 & 0.800 & 0.729 & 0.670 \\
\hline miR-145 & up & 25 & 0.859 & 0.892 & 0.743 & 0.717 & 0.666 \\
\hline miR-34a & up & 2 & 0.855 & 0.811 & 0.777 & 0.753 & 0.679 \\
\hline miR-31 & up & 5 & 0.851 & 0.840 & 0.811 & 0.739 & 0.722 \\
\hline miR-103a & up & 39 & 0.843 & 0.815 & 0.737 & 0.731 & 0.670 \\
\hline miR-487a & up & 3 & 0.839 & 0.830 & 0.720 & 0.759 & 0.685 \\
\hline miR-299 & up & 5 & 0.836 & 0.782 & 0.743 & 0.724 & 0.658 \\
\hline miR-503 & up & 6 & 0.824 & 0.830 & 0.800 & 0.714 & 0.683 \\
\hline miR-133b & up & 2 & 0.817 & 0.831 & 1.000 & 0.705 & 0.657 \\
\hline miR-150 & up & 1 & 0.811 & 0.896 & 0.806 & 0.673 & 0.720 \\
\hline miR-212 & up & 52 & 0.810 & 0.736 & 0.714 & 0.732 & 0.670 \\
\hline miR-92a & up & 8 & 0.806 & 0.774 & 0.880 & 0.727 & 0.634 \\
\hline
\end{tabular}

miRNA markers showed weak association with other cancers (besides PDAC).

\section{Conclusion}

In conclusion, we developed a novel single and multimarker identification approach for PDAC diagnosis by analyzing integrated mRNA and miRNA gene expression profiles. To overcome overfitting of imbalanced data, we applied a SVM model with sample class weights and cross-validation, based on sample partitioning in our dataset and independent datasets. Finally, we identified 705 multi-markers for 27 miRNAs and 289 genes as promising potential biomarkers for pancreatic cancer.

\section{Methods and materials}

\section{Expression profile datasets}

To identify multi-markers in pancreatic cancer, we used mRNA and miRNA expression data from 104 PDAC patients and 17 normal pancreatic patients, following surgery for kidney stones and non-malignant pancreatic disease at Seoul National University Hospital (SNUH) (The detailed experiment and pre-processing steps are described in [15]). All human subjects studies were approved by the Institutional Review Board of Seoul National University Hospital. In this dataset, mRNA and miRNA expression levels were profiled on Affymetrix (Santa Clara, CA, USA) HuGene 1.0 ST (33,297 probes) arrays and Affymetrix GeneChip miRNA 3.0 (25,016 probes) arrays, respectively. We used 5,617 human miRNA probes, out of 25,016 probes, on the Affymetrix GeneChip miRNA 3.0 array.

For validation with independent datasets of selected multi-marker candidates, we collected expression datasets for PDAC (GSE32688 [23], GSE15471 [24], and GSE16515 [25]), lymphoma (LP; GSE14879 [26]), breast cancer (BC; GSE10780 [27]), hepatocellular carcinoma (HCC; GSE6764 [28]), and lung carcinoma (LC; GSE19188 [29]) from the Gene Expression Omnibus (GEO) [30]. All collected expressed data were performed using quantile normalization and RMA normalization by $\mathrm{R}$ package. 
Table 3 Coregulated target genes.

\begin{tabular}{|c|c|c|c|}
\hline $\begin{array}{l}\text { Target } \\
\text { gene }\end{array}$ & GO & $\begin{array}{l}\text { No. of } \\
\text { miRNAs }\end{array}$ & miRNAs \\
\hline DTNA & signal transduction & 12 & $\begin{array}{l}\text { let-7i, miR-103a, miR-107, miR-135b, miR-203, miR-212, miR-21, miR-222, miR-223, miR-23a, } \\
\text { miR-299, miR-34 }\end{array}$ \\
\hline NTRK2 & Apoptosis & 11 & $\begin{array}{l}\text { let-7i, miR-103a, miR-107, miR-203, miR-212, miR-21, miR-222, miR-223, miR-23a, miR-299, } \\
\text { miR-31 }\end{array}$ \\
\hline PHF17 & Apoptosis & 11 & $\begin{array}{l}\text { let-7i, miR-103a, miR-107, miR-135b, miR-145, miR-155, miR-21, miR-212, miR-21, miR-222, } \\
\text { miR-23a }\end{array}$ \\
\hline DMD & $\begin{array}{l}\text { extracellular matrix } \\
\text { organization }\end{array}$ & 9 & let-7i, miR-103a, miR-107, miR-155, miR-203, miR-212, miR-21, miR-223, miR-31 \\
\hline SEMA6D & development & 9 & miR-103a, miR-107, miR-135b, miR-212, miR-222, miR-23a, miR-31, miR-503, miR-92a \\
\hline EPB41L4B & $\begin{array}{l}\text { actomyosin structure } \\
\text { organization }\end{array}$ & 9 & let-7i, miR-103a, miR-107, miR-203, miR-212, miR-23a, miR-31, miR-487a, miR-503 \\
\hline BCAT1 & cell cycle & 9 & let-7i, miR-135b, miR-145, miR-155, miR-196b, miR-203, miR-21, miR-28, miR-34 \\
\hline FAM13A & signal transduction & 8 & miR-203, miR-212, miR-21, miR-222, miR-223, miR-23a, miR-34, miR-487a \\
\hline GOLGA8A & & 8 & miR-100, miR-203, miR-203, miR-223, miR-223, miR-23, miR-23a, miR-92a \\
\hline ADHFE1 & metabolism & 7 & let-7i, miR-203, miR-222, miR-223, miR-23a, miR-28, miR-31 \\
\hline ARHGAP24 & angiogenesis & 7 & miR-103a, miR-107, miR-145, miR-203, miR-21, miR-223, miR-23a \\
\hline ATP8A1 & metabolism & 7 & miR-103a, miR-107, miR-135b, miR-203, miR-23a, miR-28, miR-31 \\
\hline SLC39A14 & ion transport & 7 & miR-155, miR-212, miR-222, miR-223, miR-23a, miR-28, miR-31 \\
\hline ERI2 & metabolism & 7 & let-7i, miR-100, miR-103a, miR-107, miR-203, miR-222, miR-23a \\
\hline LGR4 & immune response & 7 & let-7i, miR-203, miR-212, miR-222, miR-223, miR-23a, miR-31 \\
\hline SETBP1 & & 7 & miR-103a, miR-107, miR-135b, miR-203, miR-21, miR-223, miR-28 \\
\hline INSIG1 & cell proliferation & 7 & miR-100, miR-103a, miR-203, miR-212, miR-222, miR-34, miR-92a \\
\hline
\end{tabular}

miRNA and mRNA biomarker identification for diagnosis of pancreatic cancer

We developed a novel approach to identify candidate mRNA and miRNA multi-markers for PDAC. The schematic workflow of our pipeline is depicted in Figure 1. Paired miRNA and mRNA expression, and miRNAmRNA networks were integrated to predict performance for diagnosis of PDAC. This approach is composed of five steps. First, the relationships between miRNA and its target genes were constructed by miRNA target prediction tools. Second, mRNA and miRNA biomarker candidates were detected using our PDAC expression data. In the third step, mRNA and miRNA biomarker candidates were validated by independent datasets. Fourth, diagnostic performances of the validated marker candidates were checked in other cancers. Finally, in the last step, the biological functions of the validated marker candidates were annotated.

\section{Step 1: Prediction of miRNA-target gene interaction}

Although many miRNA studies have been performed, only a few miRNA targets have been well validated. To collect reliable miRNA-target relationships covering almost all miRNAs, we employed several in silico prediction algorithms. First, we used all validated target information for 567 miRNAs from miRTarBase 4.0 [31], and predicted target information for 2,735 miRNAs from three miRNA target prediction methods such as TargetScan version 6.2 [8],
PITA version hg18 [9], and miRvestigator [10]. These three prediction methods were evaluated as reliable methods in [32]. In this paper, we used 1,357,560 miRNA-target relationship data for 2,735 miRNAs and 18,505 targeted genes. For detecting more reliable miRNA-target relationships for specific conditions such as PDAC, only negatively correlated expressed target genes (correlation coefficient < -0.3 and p-value $<0.05$ using linear regression) were chosen (Figure 2). Finally, 33,422 miRNA-target relationship data points, for 1,176 miRNAs and 6,424 targeted genes, were used in this study.

\section{Step 2: Identification of multi-marker candidates with PDAC data}

To identify multi-marker candidates, we focused on classification performance with PDAC tissues and benign tissues. In this step, support vector machine (SVM) was applied for qualitative classification evaluated with leave-one-out cross validation (LOOCV). In consideration of our imbalanced sample size (i.e., having many more cancer than benign sample datasets), SVM was employed with sample class weights $\left(\alpha_{\text {cancer }}=1\right.$ and $\alpha_{\text {normal }}=6.117647$ ) [33]. BA, area under the curve (AUC), and p-values from the permutation tests were used for assessing the performance of each prediction model. Using LOOCV, we calculated BA and AUC values from the prediction accuracies of each marker in the testing dataset. BA is defined as an average of 


\begin{tabular}{|c|c|c|c|c|c|c|c|c|c|c|c|c|c|c|c|c|c|c|c|c|c|c|c|c|c|}
\hline Go & $\begin{array}{l}\text { target gene } \\
\end{array}$ & PC1 & BC & HCC & LC & LP & miRNA & PC1 & $B C$ & $\mathrm{HCC}$ & LC & LP & Go & target gene & PC1 & BC & HCC & LC & LP & miRNA & PC1 & BC & HCC & LC & LP \\
\hline \multirow{2}{*}{ angiogenesis } & KLF5 & 0.93 & 0.62 & 0.50 & \begin{tabular}{|l|}
0.63 \\
\end{tabular} & 0.66 & miR-148a & 0.90 & 0.63 & 0.62 & 0.65 & 0.65 & \multirow{4}{*}{$\begin{array}{l}\text { immune } \\
\text { response }\end{array}$} & VTCN1 & 0.83 & 0.50 & 0.50 & \begin{tabular}{|l|}
0.75 \\
\end{tabular} & \begin{tabular}{|l|}
0.50 \\
\end{tabular} & miR-107 & 0.80 & 0.63 & \begin{tabular}{|l|l|}
0.63 \\
\end{tabular} & \begin{tabular}{|l}
0.63 \\
\end{tabular} & 0.62 \\
\hline & \begin{tabular}{|l|} 
PTPN14 \\
\end{tabular} & 1.00 & 0.50 & 0.50 & \begin{tabular}{|l|}
0.62 \\
\end{tabular} & 0.48 & miR-148a & 0.90 & 0.63 & 0.62 & 0.65 & 0.65 & & PELI2 & 1.00 & 0.50 & 0.62 & \begin{tabular}{|l|}
0.76 \\
\end{tabular} & \begin{tabular}{|l|}
0.50 \\
\end{tabular} & miR-135b & 0.87 & 0.62 & \begin{tabular}{|l|l|}
0.64 \\
\end{tabular} & \begin{tabular}{|l}
0.68 \\
\end{tabular} & 0.63 \\
\hline \multirow{19}{*}{ Apotosis } & KIAA1324 & 0.92 & 0.50 & 0.50 & 0.54 & 0.75 & miR-107 & 0.80 & 0.63 & 0.63 & 0.63 & 0.62 & & $c 7$ & 0.83 & 0.50 & 0.50 & 0.89 & 0.75 & miR-21 & 0.90 & 0.63 & 0.63 & 0.64 & 0.63 \\
\hline & BTG2 & 0.80 & 0.50 & 0.50 & \begin{tabular}{|l|}
0.78 \\
\end{tabular} & 0.83 & miR-107 & 0.80 & 0.63 & 0.63 & 0.63 & 0.62 & & LGR4 & 0.95 & 0.50 & 0.50 & 0.81 & \begin{tabular}{|l|}
0.61 \\
\end{tabular} & miR-31 & 0.84 & 0.63 & 0.63 & \begin{tabular}{|l}
0.67 \\
\end{tabular} & 0.63 \\
\hline & NTRK2 & 0.82 & 0.88 & 0.52 & 0.72 & 0.69 & miR-107 & 0.80 & 0.63 & 0.63 & 0.63 & $0.62 \mid$ & \multirow{10}{*}{ metabolism } & ATP8A1 & 1.00 & 0.50 & 0.50 & \begin{tabular}{|l|}
0.86 \\
\end{tabular} & \begin{tabular}{|l|l|}
0.62 \\
\end{tabular} & miR-107 & 0.80 & 0.63 & 0.63 & 0.63 & 0.62 \\
\hline & SGK1 & 0.82 & 0.50 & 0.50 & 0.74 & 0.49 & miR-107 & 0.80 & 0.63 & 0.63 & 0.63 & 0.62 & & DHRS12 & 0.84 & 0.50 & 0.71 & $|0.77|$ & 0.64 & miR-107 & 0.80 & 0.63 & 0.63 & 0.63 & 0.62 \\
\hline & PHF17 & 0.95 & 0.50 & 0.50 & 0.76 & 0.78 & miR-107 & 0.80 & \begin{tabular}{|l|l}
0.63 \\
\end{tabular} & 0.63 & 0.63 & 0.62 & & ERI2 & 0.90 & 0.50 & 0.86 & 0.73 & \begin{tabular}{|l|l|}
0.50 \\
\end{tabular} & miR-107 & 0.80 & 0.63 & \begin{tabular}{|l|}
0.63 \\
\end{tabular} & 0.63 & 0.62 \\
\hline & CLU & 0.91 & 0.71 & 0.79 & 0.88 & 0.84 & miR-107 & 0.80 & 0.63 & 0.63 & 0.63 & 0.62 & & RPLS & 1.00 & 0.50 & 0.47 & \begin{tabular}{|l|}
0.58 \\
\end{tabular} & 0.69 & miR-107 & 0.80 & 0.63 & 0.63 & 0.63 & 0.62 \\
\hline & MKNK1 & 1.00 & 0.50 & 0.50 & \begin{tabular}{|l|}
0.77 \\
\end{tabular} & 0.55 & miR-135b & 0.87 & 0.62 & 0.64 & 0.68 & 0.63 & & ATP8A1 & 1.00 & 0.50 & 0.50 & 0.86 & \begin{tabular}{|l|l|}
0.62 \\
\end{tabular} & miR-135b & 0.87 & 0.62 & 0.64 & 0.68 & 0.63 \\
\hline & PHF17 & 1.00 & 0.50 & 0.50 & 0.76 & 0.78 & miR-135b & 0.87 & 0.62 & 0.64 & 0.68 & 0.63 & & SH3PXD2A & 0.81 & 0.50 & 0.50 & \begin{tabular}{|l|}
0.64 \\
\end{tabular} & \begin{tabular}{l|l}
0.63 \\
\end{tabular} & miR-148a & 0.90 & 0.63 & 0.62 & 0.65 & 0.65 \\
\hline & SULF1 & 0.80 & 0.50 & 0.50 & \begin{tabular}{|l|} 
\\
\end{tabular} & 0.77 & miR-148a & 0.90 & 0.63 & 0.62 & 0.65 & 0.65 & & DNAJB99 & 0.81 & 0.54 & 0.50 & \begin{tabular}{|l|}
0.59 \\
\end{tabular} & \begin{tabular}{|l|}
0.59 \\
\end{tabular} & miR-21 & 0.90 & 0.63 & \begin{tabular}{|l|l|}
0.63 \\
\end{tabular} & \begin{tabular}{|l}
0.64 \\
\end{tabular} & $\mid 0.63$ \\
\hline & ADAM17 & 0.97 & 0.50 & 0.50 & 0.69 & 0.66 & miR-148a & 0.90 & 0.63 & 0.62 & 0.65 & 0.65 & & ATP8A1 & 1.00 & 0.50 & 0.50 & \begin{tabular}{|l|}
0.86 \\
\end{tabular} & \begin{tabular}{|l|}
0.62 \\
\end{tabular} & miR-31 & 0.81 & 0.61 & 0.62 & \begin{tabular}{|l}
0.66 \\
\end{tabular} & 0.65 \\
\hline & BTG2 & 0.80 & 0.50 & 0.50 & \begin{tabular}{|l|} 
\\
\end{tabular} & 0.83 & miR-21 & 0.90 & 0.63 & 0.63 & 0.64 & 0.63 & & ATP8A1 & 1.00 & 0.50 & 0.50 & \begin{tabular}{|l|}
0.86 \\
\end{tabular} & \begin{tabular}{|l|}
0.62 \\
\end{tabular} & miR-31 & 0.84 & 0.63 & 0.63 & \begin{tabular}{|l|l|} 
& 0.67 \\
\end{tabular} & \begin{tabular}{|l|l|}
0.63 \\
\end{tabular} \\
\hline & NTRK2 & 0.82 & 0.88 & 0.52 & \begin{tabular}{|l|}
0.72 \\
\end{tabular} & 0.69 & miR-21 & 0.90 & 0.63 & 0.63 & 0.64 & 0.63 & & DNAJB9 & 0.81 & 0.54 & 0.50 & \begin{tabular}{|l|}
0.59 \\
\end{tabular} & \begin{tabular}{|l|l|}
0.59 \\
\end{tabular} & miR-92 & 0.88 & 0.63 & 0.62 & \begin{tabular}{|l|l|} 
& 0.67 \\
\end{tabular} & $\mid 0.63$ \\
\hline & PHF17 & 0.95 & 0.50 & 0.50 & \begin{tabular}{|l|}
0.76 \\
\end{tabular} & 0.78 & miR-21 & 0.90 & 0.63 & 0.63 & 0.64 & 0.63 & \multirow{11}{*}{$\begin{array}{l}\text { regulation of } \\
\text { transcription }\end{array}$} & MYCL1 & 0.86 & 0.50 & 0.50 & 0.71 & \begin{tabular}{|l|l|}
0.63 \\
\end{tabular} & miR-107 & 0.80 & 0.63 & \begin{tabular}{|l|l|}
0.63 \\
\end{tabular} & \begin{tabular}{|l}
0.63 \\
\end{tabular} & $\mid 0.62$ \\
\hline & PDE3A & 0.96 & 0.50 & 0.50 & 0.58 & 0.82 & miR-21 & 0.90 & 0.63 & 0.63 & 0.64 & 0.63 & & VLDLR & 1.00 & 0.50 & 0.71 & \begin{tabular}{|l|}
0.67 \\
\end{tabular} & \begin{tabular}{|l|l|l|l|}
0.66 \\
\end{tabular} & miR-135b & 0.87 & 0.62 & 0.64 & 0.68 & 0.63 \\
\hline & CXCL12 & 0.85 & 0.50 & 0.78 & 0.82 & 0.93 & miR-31 & 0.84 & 0.63 & 0.63 & 0.67 & 0.63 & & NR3C2 & 1.00 & 0.50 & 0.67 & 0.89 & 0.50 & miR-135b & 0.87 & 0.62 & 0.64 & 0.68 & 0.63 \\
\hline & KIAA1324 & 0.92 & 0.50 & 0.50 & 0.54 & 0.75 & miR-31 & 0.84 & 0.63 & 0.63 & 0.67 & 0.63 & & TRAK1 & 0.86 & 0.67 & 0.50 & \begin{tabular}{|l|}
0.86 \\
\end{tabular} & \begin{tabular}{|c|}
0.55 \\
\end{tabular} & miR-148a & 0.90 & 0.63 & 0.62 & 0.65 & 0.65 \\
\hline & NTRK2 & 0.82 & 0.88 & 0.52 & 0.72 & 0.69 & miR-31 & 0.84 & 0.63 & 0.63 & 0.67 & 0.63 & & ETV1 & 1.00 & 0.50 & 0.50 & 0.87 & \begin{tabular}{l|l|l|l|}
0.63 \\
\end{tabular} & \begin{tabular}{|l|} 
miR-148a \\
\end{tabular} & 0.90 & 0.63 & 0.62 & 0.65 & 0.65 \\
\hline & SGK1 & 0.82 & 0.50 & 0.50 & \begin{tabular}{|l|}
0.74 \\
\end{tabular} & 0.49 & miR-503 & 0.80 & \begin{tabular}{|l|l|}
0.64 \\
\end{tabular} & 0.65 & 0.60 & 0.59 & & MET & 1.00 & 0.50 & 0.50 & 0.65 & \begin{tabular}{|l|l|}
0.66 \\
\end{tabular} & miR-148a & 0.90 & 0.63 & \begin{tabular}{|l|}
0.62 \\
\end{tabular} & 0.65 & 0.65 \\
\hline & BTG2 & 0.80 & 0.50 & 0.50 & 0.78 & 0.83 & miR.92 & 0.88 & 0.63 & 0.62 & 0.67 & 0.63 & & \begin{tabular}{|l|} 
MYCL1 \\
\end{tabular} & 0.86 & 0.50 & 0.50 & 0.71 & 0.63 & miR-21 & 0.90 & 0.63 & 0.63 & 0.64 & 0.63 \\
\hline \multirow{7}{*}{ biosynthesis } & TKT & 0.91 & 0.50 & 0.50 & 0.60 & 0.75 & \begin{tabular}{||l|} 
miR-107 \\
\end{tabular} & 0.80 & 0.63 & 0.63 & 0.63 & 0.62 & & PBX1 & 0.83 & 0.50 & 0.50 & 0.76 & \begin{tabular}{|l|l|}
0.49 \\
\end{tabular} & miR-21 & 0.90 & 0.63 & 0.63 & 0.64 & 0.63 \\
\hline & \begin{tabular}{|l|} 
ALDH1L1 \\
\end{tabular} & 0.85 & 0.50 & 0.50 & 0.56 & 0.48 & miR-107 & 0.80 & 0.63 & 0.63 & 0.63 & 0.62 & & RBPJ & 0.85 & 0.50 & 0.00 & 0.58 & 0.58 & miR-31 & 0.84 & 0.63 & 0.63 & 0.67 & 0.63 \\
\hline & ALDH1L1 & 0.85 & 0.50 & 0.50 & \begin{tabular}{|l|}
0.56 \\
\end{tabular} & 0.48 & miR-107 & 0.80 & 0.63 & 0.63 & 0.63 & 0.62 & & MYCL1 & 0.86 & 0.50 & 0.50 & \begin{tabular}{|l|}
0.71 \\
\end{tabular} & \begin{tabular}{l|l}
0.63 \\
\end{tabular} & miR-34a & 0.81 & 0.63 & 0.64 & 0.68 & 0.63 \\
\hline & SLC2A1 & 0.91 & 0.50 & 0.50 & 0.90 & 0.78 & miR-148a & 0.90 & 0.63 & 0.62 & 0.65 & 0.65 & & \begin{tabular}{|l|} 
INSR \\
\end{tabular} & 0.80 & 0.50 & 0.50 & 0.58 & 0.50 & miR-503 & 0.80 & 0.64 & 0.65 & 0.60 & 0.59 \\
\hline & MBOAT2 & 0.93 & 0.50 & 0.50 & 0.73 & 0.63 & miR-148a & 0.90 & 0.63 & 0.62 & 0.65 & 0.65 & \multirow{8}{*}{$\begin{array}{c}\text { signal } \\
\text { transduction }\end{array}$} & DTNA & 0.94 & 0.50 & 0.92 & 0.77 & 0.75 & miR-107 & 0.80 & 0.63 & 0.63 & 0.63 & 0.62 \\
\hline & $\operatorname{scD} 5$ & 0.85 & 0.50 & 0.50 & \begin{tabular}{|l|}
0.75 \\
\end{tabular} & 0.82 & miR-21 & 0.90 & 0.63 & 0.63 & 0.64 & 0.63 & & DTNA & 1.00 & 0.50 & \begin{tabular}{|l|}
0.92 \\
\end{tabular} & \begin{tabular}{|l|}
0.77 \\
\end{tabular} & \begin{tabular}{|l|}
0.75 \\
\end{tabular} & miR-135b & 0.87 & 0.62 & \begin{tabular}{|l|l|}
0.64 \\
\end{tabular} & \begin{tabular}{|l}
0.68 \\
\end{tabular} & \begin{tabular}{|l}
0.63 \\
\end{tabular} \\
\hline & TKT & 0.91 & 0.50 & 0.50 & 0.60 & 0.75 & miR-31 & 0.84 & 0.63 & 0.63 & 0.67 & 0.63 & & RAB34 & 0.90 & 0.50 & 0.50 & 0.65 & \begin{tabular}{|l|}
0.77 \\
\end{tabular} & miR-148a & 0.90 & 0.63 & \begin{tabular}{|l|l|}
0.62 \\
\end{tabular} & 0.65 & \begin{tabular}{|l}
0.65 \\
\end{tabular} \\
\hline \multirow{7}{*}{ cell cycle } & USP2 & 0.96 & 0.50 & 0.50 & 0.62 & 0.50 & \begin{tabular}{|l|} 
miR-107 \\
\end{tabular} & 0.80 & 0.63 & 0.63 & 0.63 & $\begin{array}{ll}0.62 \\
\end{array}$ & & DTNA & 0.94 & 0.50 & 0.92 & \begin{tabular}{|l|}
0.77 \\
\end{tabular} & 0.75 & miR-21 & 0.90 & 0.63 & 0.63 & 0.64 & 0.63 \\
\hline & BCAI1 & 1.00 & 0.50 & 0.85 & \begin{tabular}{|l|}
0.61 \\
\end{tabular} & 0.72 & miR-135b & 0.87 & 0.62 & 0.64 & 0.68 & \begin{tabular}{|l|}
0.63 \\
\end{tabular} & & KSR1 & 1.00 & 0.50 & 0.60 & \begin{tabular}{|l|}
0.56 \\
\end{tabular} & \begin{tabular}{|l|l|}
0.66 \\
\end{tabular} & miR-222 & 0.87 & 0.61 & \begin{tabular}{|l|l|}
0.74 \\
\end{tabular} & 0.70 & 0.72 \\
\hline & CDC25B & 0.80 & 0.62 & 0.50 & \begin{tabular}{|l|} 
\\
\end{tabular} & 0.48 & miR-148a & 0.90 & 0.63 & 0.62 & 0.65 & \begin{tabular}{|l|}
0.65 \\
\end{tabular} & & GLP1R & 0.89 & 0.50 & 0.69 & \begin{tabular}{|l|}
0.65 \\
\end{tabular} & \begin{tabular}{|l|}
0.68 \\
\end{tabular} & miR-31 & 0.84 & 0.63 & 0.63 & \begin{tabular}{|l}
0.67 \\
\end{tabular} & 0.63 \\
\hline & BCAI1 & 0.90 & 0.50 & 0.85 & \begin{tabular}{|l|}
0.61 \\
\end{tabular} & 0.72 & miR-21 & 0.90 & \begin{tabular}{|l|}
0.63 \\
\end{tabular} & 0.63 & $\mid 0.64$ & \begin{tabular}{|l|}
0.63 \\
\end{tabular} & & KSR1 & 1.00 & 0.50 & 0.60 & \begin{tabular}{|l|}
0.56 \\
\end{tabular} & \begin{tabular}{|l|l|}
0.66 \\
\end{tabular} & miR-31 & 0.84 & 0.63 & \begin{tabular}{|l|l|}
0.63 \\
\end{tabular} & \begin{tabular}{|l}
0.67 \\
\end{tabular} & 0.63 \\
\hline & USP2 & 0.96 & 0.50 & 0.50 & \begin{tabular}{|l|}
0.62 \\
\end{tabular} & 0.50 & miR-31 & 0.84 & 0.63 & 0.63 & 0.67 & 0.63 & & DTNA & 0.94 & 0.50 & 0.92 & \begin{tabular}{|l|}
0.77 \\
\end{tabular} & \begin{tabular}{|l|l|} 
\\
\end{tabular} & miR-34a & 0.81 & 0.63 & 0.64 & 0.68 & 0.63 \\
\hline & BCAI1 & 0.90 & 0.50 & 0.85 & 0.61 & 0.72 & miR-34a & 0.81 & 0.63 & 0.64 & \begin{tabular}{|l|l|} 
& 0.68
\end{tabular} & 0.63 & $\begin{array}{l}\text { gnaling } \\
\text { athway }\end{array}$ & EIF4B & 1.00 & 0.50 & 0.50 & 0.63 & \begin{tabular}{|l|}
0.66 \\
\end{tabular} & miR-150 & 0.81 & 0.61 & 0.58 & 0.58 & 0.58 \\
\hline & USP2 & 0.96 & 0.50 & 0.50 & 0.62 & 0.50 & miR-503 & 0.80 & 0.64 & 0.65 & 0.60 & 0.59 & & SETBP1 & 0.82 & 0.50 & 0.50 & 0.75 & \begin{tabular}{|l|l}
0.62 \\
\end{tabular} & miR-107 & 0.80 & 0.63 & 0.63 & 0.63 & 0.62 \\
\hline \multirow{4}{*}{$\begin{array}{c}\text { cell } \\
\text { proliferation }\end{array}$} & LIFR & 0.90 & 0.91 & 1.00 & 0.88 & 0.82 & miR-21 & 0.90 & 0.63 & 0.63 & 0.64 & 0. & & C9orf41 & 0.86 & 0.50 & 0.50 & 0.00 & 0.50 & \begin{tabular}{|l|l} 
miR-107 \\
\end{tabular} & 0.80 & 0.63 & 0.63 & 0.63 & 0.62 \\
\hline & UFFR & 0.90 & 0.91 & 1.00 & 0.88 & 0.82 & mik-31 & 0.84 & 0.63 & 0.63 & 0.67 & 0.63 & & \begin{tabular}{|l|} 
C140rf45 \\
\end{tabular} & 0.86 & 0.50 & 0.72 & 0.58 & \begin{tabular}{|l|}
0.50 \\
\end{tabular} & miR-107 & 0.80 & 0.63 & 0.63 & 0.63 & 0.62 \\
\hline & \begin{tabular}{|l|} 
INSIG1 \\
\end{tabular} & 0.90 & 0.50 & 0.50 & \begin{tabular}{|l|} 
\\
\end{tabular} & 0.87 & miR-34a & 0.81 & 0.63 & 0.64 & 0.68 & 0.63 & & FAM171A1 & 0.85 & 0.82 & 0.50 & \begin{tabular}{|l|}
0.65 \\
\end{tabular} & \begin{tabular}{|l|l|}
0.56 \\
\end{tabular} & miR-107 & 0.80 & 0.63 & \begin{tabular}{|l|}
0.63 \\
\end{tabular} & \begin{tabular}{|l}
0.63 \\
\end{tabular} & 0.62 \\
\hline & \begin{tabular}{|l|} 
INSIG1 \\
\end{tabular} & 0.90 & 0.50 & 0.50 & 0.76 & 0.87 & miR-92 & 0.88 & 0.63 & 0.62 & 0.67 & 0.63 & & BACE1 & 1.00 & 0.50 & 0.50 & 0.67 & 0.62 & miR-135b & 0.87 & 0.62 & 0.64 & 0.68 & 0.63 \\
\hline & IFRD1 & 0.95 & 0.50 & 0.50 & \begin{tabular}{|l|}
0.50 \\
\end{tabular} & 0.50 & miR-107 & 0.80 & 0.63 & 0.63 & 0.63 & 0.62 & & RRBP1 & 1.00 & 0.50 & 0.50 & \begin{tabular}{|l|}
0.57 \\
\end{tabular} & \begin{tabular}{|c|}
0.77 \\
\end{tabular} & miR-135b & 0.87 & 0.62 & \begin{tabular}{|l|}
0.64 \\
\end{tabular} & 0.68 & \begin{tabular}{|l}
0.63 \\
\end{tabular} \\
\hline & SEMA6D & 1.00 & 0.52 & 0.69 & 0.63 & 0.55 & miR-135b & \begin{tabular}{|l|l|}
0.87 \\
\end{tabular} & 0.62 & 0.64 & 0.68 & 0.63 & & SETBP1 & 1.00 & 0.50 & 0.50 & 0.75 & 0.62 & miR-135b & 0.87 & 0.62 & 0.64 & 0.68 & 0.63 \\
\hline & CTTNBP2 & 1.00 & 0.50 & 0.43 & 0.00 & 0.49 & miR-135b & 0.87 & 0.62 & 0.64 & 0.68 & 0.63 & & LRCH1 & 0.91 & 0.50 & 0.50 & \begin{tabular}{|l|}
0.57 \\
\end{tabular} & \begin{tabular}{|l|l|}
0.50 & \\
\end{tabular} & miR-148a & 0.90 & 0.63 & 0.62 & 0.65 & 0.65 \\
\hline development & \begin{tabular}{|l|} 
IFRD1 \\
\end{tabular} & 0.95 & 0.50 & 0.50 & 0.50 & 0.50 & miR-21 & 0.90 & 0.63 & 0.63 & 0.64 & 0.63 & Others & TMSB10 & 0.88 & 0.50 & 0.43 & 0.40 & 0.88 & \begin{tabular}{|l|} 
miR-148a \\
\end{tabular} & 0.90 & 0.63 & 0.62 & 0.65 & 0.65 \\
\hline & ACAI1 & 1.00 & 0.50 & 0.74 & 0.77 & 0.61 & miR-21 & 0.90 & 0.63 & 0.63 & 0.64 & 0.63 & & SNTB1 & 1.00 & 0.54 & 0.50 & 0.77 & 0.50 & miR-21 & 0.90 & 0.63 & 0.63 & 0.64 & 0.63 \\
\hline & \begin{tabular}{|l|} 
ACAI1 \\
\end{tabular} & 1.00 & 0.50 & 0.74 & \begin{tabular}{|l|}
0.77 \\
\end{tabular} & 0.61 & miR-31 & 0.84 & 0.63 & 0.63 & 0.67 & 0.63 & & DCAF11 & 0.92 & 0.50 & 0.82 & 0.55 & \begin{tabular}{|l|l|}
0.47 \\
\end{tabular} & miR-21 & 0.90 & 0.63 & 0.63 & 0.64 & 0.63 \\
\hline & STOX2 & 0.81 & 0.50 & 0.50 & 0.00 & 0.56 & |miR-503 & 0.80 & 0.64 & 0.65 & 0.60 & 0.5 & & SETBP1 & 0.82 & 0.50 & 0.50 & 0.75 & \begin{tabular}{l|l}
0.62 \\
\end{tabular} & miR-21 & 0.90 & 0.63 & 0.63 & 0.64 & 0.63 \\
\hline & DMD & 0.82 & 0.52 & 0.50 & 0.85 & 0.72 & |miR-107 & 0.80 & 0.63 & 0.63 & 0.63 & 0.62 & & ZNF704 & 1.00 & 0.52 & 0.50 & 0.00 & \begin{tabular}{|l|}
0.58 \\
\end{tabular} & miR-21 & 0.90 & 0.63 & 0.63 & 0.64 & 0.63 \\
\hline & DMD & 0.82 & 0.52 & 0.50 & \begin{tabular}{|l|}
0.85 \\
\end{tabular} & 0.72 & miR-107 & 0.80 & 0.63 & 0.63 & 0.63 & \begin{tabular}{|l|} 
\\
\end{tabular} & & ZNF704 & 1.00 & 0.52 & 0.50 & \begin{tabular}{|l|}
0.00 \\
\end{tabular} & \begin{tabular}{|l|}
0.58 \\
\end{tabular} & miR-31 & 0.81 & 0.61 & \begin{tabular}{|l|l|}
0.62 \\
\end{tabular} & \begin{tabular}{|l}
0.66 \\
\end{tabular} & 0.65 \\
\hline & LTBP1 & 0.81 & 0.50 & $\begin{array}{ll}0.47 \\
\end{array}$ & 0.62 & 0.81 & miR-148a & 0.90 & 0.63 & 0.62 & 0.65 & 0.65 & & SLC41A1 & 0.89 & 0.50 & 0.50 & \begin{tabular}{|l|}
0.77 \\
\end{tabular} & 0.50 & miR-31 & 0.84 & 0.63 & 0.63 & 0.67 & 0.63 \\
\hline & FBN1 & 0.91 & 0.50 & 0.50 & \begin{tabular}{|l|}
0.49 \\
\end{tabular} & 0.76 & $\mathrm{mi}$ & 0.90 & 0.63 & 0.62 & 0.65 & 0.6 & & ZNF704 & 1.00 & 0.52 & \begin{tabular}{|l|l|} 
\\
\end{tabular} & \begin{tabular}{|l|}
0.00 \\
\end{tabular} & \begin{tabular}{|l|}
0.58 \\
\end{tabular} & miR-31 & 0.84 & 0.63 & \begin{tabular}{|l|l|}
0.63 \\
\end{tabular} & \begin{tabular}{|l|l}
0.67 \\
\end{tabular} & 0.63 \\
\hline & DMD & 0.82 & 0.52 & 0.50 & 0.85 & 0.72 & miR-21 & 0.90 & 0.63 & 0.63 & 0.64 & 0.63 & & GOLGABA & 0.91 & 0.50 & 0.49 & \begin{tabular}{|l|} 
\\
\end{tabular} & \begin{tabular}{|l|l|}
0.49 \\
\end{tabular} & \begin{tabular}{|l|l|} 
miR-92 \\
\end{tabular} & 0.88 & 0.63 & 0.62 & 0.67 & 0.63 \\
\hline & DMD & 0.82 & 0.52 & 0.50 & 0.85 & 0.72 & miR-31 & 0.81 & \begin{tabular}{|l|l|} 
& 0.61 \\
\end{tabular} & 0.62 & 0.66 & & & & & & & & & & & & & & \\
\hline & DMD & 0.82 & 0.52 & 0.50 & 0.85 & 0.72 & miR-31 & 0.84 & \begin{tabular}{|l|l|}
0.63 \\
\end{tabular} & 0.63 & 0.67 & 0.63 & & & & & & & & & & & & & \\
\hline
\end{tabular}

Figure 3 miRNA-target gene network and Gene ontology. Blue diamond is miRNA. Circle node is gene. Red circle node is gene with gene ontology related with cancerization such as apoptosis, angiogenesis, cell proliferation, blood vessel development, transcriptional regulation, and immune response.

sensitivity and specificity, and is a more appropriate evaluation measure for imbalanced datasets than conventional accuracy (i.e., the proportion of the true results among the number of total test datasets). The permutation p-values were calculated from empirical null distribution of BAs by $1 \times 10^{6}$ sample permutations for markers with high BAs.

Using the miRNA and mRNA target relationships generated in step 1, 1504 multi-markers for 217 genes and 56 miRNAs were selected with BAs $>0.8$, AUC $>0.8$, and Bonferroni adjusted p-values $<0.05$ for genes and miRNAs, respectively.

\section{Step 3: Evaluation of prediction performance in independent PDAC datasets}

To avoid selection of markers with specific data-dependency or specific platform-dependency, all identified single or multi-markers were evaluated using three public, independent PDAC datasets collected from the GEO [30] (Table 2). Of the three, PDAC dataset1 had both 


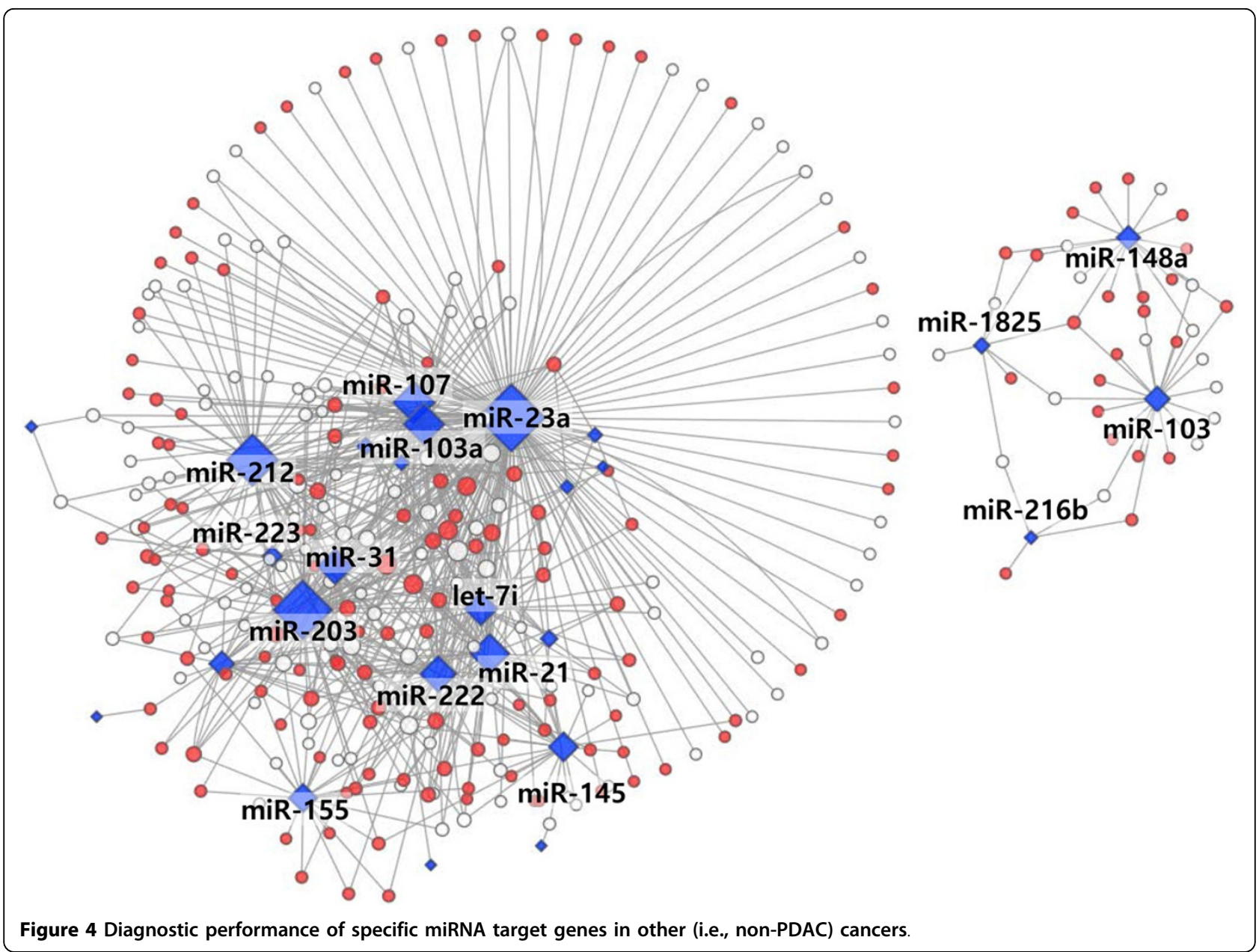

mRNA and miRNA expression microarray profiles from GSE32688 [23], while PDAC dataset2 and dataset3 had only mRNA expression profiles using microarray data from GSE15471 [14] and GSE16515 [25]. To select reliable and robust miRNA-target gene multi-markers, miRNAs and their putative target genes having negatively correlated expression, and BAs $>0.7$ in PDAC dataset1, were selected.

To validate miRNA prediction performance in the profile datasets (PDAC datasets 2 and 3) containing only mRNA expression, we estimated the expression of specific miRNAs using their predicted miRNA-target gene relationships. In Figure 2, linear regression models were fitted with miRNA and mRNA expression data from the 104 cancer tissues and 17 benign tissues. Then, the expression of the miRNAs of interest was estimated by regression models and its targeted-gene expression data in the independent datasets. Using this estimated miRNA expression, its prediction performance could then be calculated. We extracted the multi-markers with BAs $>0.7$ in one or more of the PDAC datasets 2 and/or 3 . Finally, after validation with the three independent PDAC datasets, we selected 712 miRNAtarget gene multi-markers for 30 miRNAs and 290 genes.

\section{Step 4: Evaluation of prediction performance in other cancer datasets}

To examine the feasibility of repurposing our identified marker candidates for other cancers, we collected other cancer datasets having mRNA expression data for lymphoma [26], breast cancer [27], hepatocellular carcinoma [28], and lung carcinoma [29] from GEO datasets. Based on SVM-LOOCV evaluation analysis, the selected single and multi-markers were evaluated.

\section{Step 5: Gene ontology analysis and miRNA-mRNA} network generation using the identified biomarkers The targeted genes of the identified multi-markers were annotated for gene ontology pathways/processes (GO) using PANTHER [34]. In this analysis, markers with annotation results with Bonferroni-corrected p-values < 0.05 were selected. Using this GO annotation, miRNAtarget gene relationships of identified multi-markers 
were represented by the network generated by Cytoscape 3.1.1 [35] (Figure 3).

\section{List of abbreviations used}

AUC, Area under curve; BA, Balanced accuracy; BR, Breast cancer; GEO, Gene Expression Omnibus; GO, Gene ontology; HCC, Hepatocellular carcinoma; LC, Lung cancer; LOOCV, Leave-one-out cross-validation; LP, Lymphoma; mRNA, messenger RNA; miRNA, microRNA; PDAC, Pancreatic ductal

adenocarcinoma; SVM, Support vector machine; TCGA, the Cancer Genome Atlas;

\section{Competing interests}

The authors declare that they have no competing interests.

\section{Authors' contributions}

MK performed the analysis, and drafted the manuscript. YK performed the analysis of microarray. SL participated in the design of the study. JN, TY, SY and SH performed the microarray experiment. MK, SK and JJ conducted the sample collection and preparation. TP and JJ conceived of the study, and participated in its design and coordination. TP helped to draft the manuscript. All authors write, read and approved the final manuscript.

\section{Acknowledgements}

Publication of this work was supported by the National Research Foundation of Korea (NRF) grant funded by the Korean government (MSIP) (2012R1A3A2026438, 2013M3A9C4078158, 2013R1A1A3010025) and Healthcare Group, Future Technology R\&D Division, SK telecom Co. This article has been published as part of BMC Genomics Volume 16 Supplement 9, 2015: Selected articles from the IEE International Conference on Bioinformatics and Biomedicine (BIBM 2014): Genomics. The full contents of the supplement are available online at http://www.biomedcentral.com/ bmcgenomics/supplements/16/S9.

\section{Authors' details}

'Interdisciplinary program in Bioinformatics, Seoul National University, Seoul, Korea. ${ }^{2}$ Department of Statistics, Seoul National University, Seoul, Korea. ${ }^{3}$ Department of Mathematics and Statistics, Sejong University, Seoul, Korea. ${ }^{4}$ Immunodiagnostics R\&D Team, IVD Business Unit, New Business Division, SK telecom Co., Seongnam, Korea. ${ }^{5}$ Department of Surgery, Seoul National University Hospital, Seoul, Korea.

\section{Published: 17 August 2015}

\section{References}

1. Bartel DP, Chen CZ: Micromanagers of gene expression: the potentially widespread influence of metazoan microRNAs. Nature Reviews Genetics 2004, 5(5):396-400.

2. Johnson CD, Esquela-Kerscher A, Stefani G, Byrom M, Kelnar K, Ovcharenko $D$, et al: The let-7 microRNA represses cell proliferation pathways in human cells. Cancer Research 2007, 67(16):7713-7722.

3. Hermeking H: The miR-34 family in cancer and apoptosis. Cell Death Diff 2010, 17(2):193-199.

4. Kuehbacher A, Urbich C, Dimmeler S: Targeting microRNA expression to regulate angiogenesis. Trends Pharmacol 2008, 29(1):12-15.

5. Nicoloso MS, Spizzo R, Shimizu M, Rossi S, Calin GA: MicroRNAs-the micro steering wheel of tumour metastases. Nature Reviews Cancer 2009, 9(4):293-302.

6. Bhutia YD, Hung SW, Krentz M, Patel D, Lovin D, Manoharan R, et al: Differential processing of let-7a precursors influences RRM2 expression and chemosensitivity in pancreatic cancer: role of LIN-28 and SET oncoprotein. PLoS One 2013, 8(1):e53436.

7. Srivastava SK, Arora S, Singh S, Bhardwaj A, Averett C, Singh AP: MicroRNAs in pancreatic malignancy: progress and promises. Cancer Letters 2014, 347(2):167-174.

8. Lewis $B P$, Burge $C B$, Bartel DP: Conserved seed pairing, often flanked by adenosines, indicates that thousands of human genes are microRNA targets. Cell 2005, 120(1):15-20.
9. Kertesz M, lovino N, Unnerstall U, Gaul U, Segal E: The role of site accessibility in microRNA target recognition. Nature Genetics 2007, 39(10):1278-1284.

10. Plaisier $\mathrm{CL}$, Bare JC, Baliga NS: miRvestigator: web application to identify miRNAs responsible for co-regulated gene expression patterns discovered through transcriptome profiling. Nucleic Acids Research 2011, 39(Web Server issue):W125-W131.

11. Rajewsky N: microRNA target predictions in animals. Nature genetics 2006, 38 Suppl: S8-S13.

12. Le HS, Bar-Joseph Z: Integrating sequence, expression and interaction data to determine condition-specific miRNA regulation. Bioinformatics 2013, 29(13):i89-i97.

13. Chan A, Diamandis EP, Blasutig IM: Strategies for discovering novel pancreatic cancer biomarkers. Journal of Proteomics 2013, 81:126-134

14. Hezel AF, Kimmelman AC, Stanger BZ, Bardeesy N, Depinho RA: Genetics and biology of pancreatic ductal adenocarcinoma. Genes Dev 2006, 20(10):1218-1249.

15. Kwon MS, Kim Y, Lee S, Namkung J, Yun T, Yi SG, et al: Biomarker development for pancreatic ductal adenocarcinoma using integrated analysis of mRNA and miRNA expression. Bioinformatics and Biomedicine (BIBM), 2014 IEEE International Conference 2014, 273-278.

16. Lee KH, Lotterman C, Karikari C, Omura N, Feldmann G, Habbe N, et al: Epigenetic silencing of MicroRNA miR-107 regulates cyclin-dependent kinase 6 expression in pancreatic cancer. Pancreatology 2009, 9(3):293-301.

17. Munding JB, Adai AT, Maghnouj A, Urbanik A, Zollner H, Liffers ST, et al: Global microRNA expression profiling of microdissected tissues identifies miR-135b as a novel biomarker for pancreatic ductal adenocarcinoma. Int J Cancer 2012, 131(2):E86-E95.

18. Valeri N, Braconi C, Gasparini P, Murgia C, Lampis A, Paulus-Hock V, et al: MicroRNA-135b promotes cancer progression by acting as a downstream effector of oncogenic pathways in colon cancer. Cancer Cell 2014, 25(4):469-483.

19. Xu WG, Shang YL, Cong XR, Bian X, Yuan Z: MicroRNA-135b promotes proliferation, invasion and migration of osteosarcoma cells by degrading myocardin. Int J Oncol 2014, 45(5):2024-2032.

20. Xu Q, Liu LZ, Yin Y, He J, Li Q, Qian X, et al: Regulatory circuit of PKM2/ NF-kappaB/miR-148a/152-modulated tumor angiogenesis and cancer progression. Oncogene 2015.

21. Garzon R, Marcucci G, Croce CM: Targeting microRNAs in cancer: rationale, strategies and challenges. Nature Rev Drug Discov 2010, 9(10):775-789.

22. Greither T, Grochola LF, Udelnow A, Lautenschlager C, Wurl P, Taubert H: Elevated expression of microRNAs 155, 203, 210 and 222 in pancreatic tumors is associated with poorer survival. Int I Cancer 2010, 126(1):73-80.

23. Donahue TR, Tran LM, Hill R, Li Y, Kovochich A, Calvopina JH, et al: Integrative survival-based molecular profiling of human pancreatic cancer. Clin Cancer Res 2012, 18(5):1352-1363.

24. Badea L, Herlea V, Dima SO, Dumitrascu T, Popescu I: Combined gene expression analysis of whole-tissue and microdissected pancreatic ductal adenocarcinoma identifies genes specifically overexpressed in tumor epithelia. Hepatogastroenterology 2008, 55(88):2016-2027.

25. Pei H, Li L, Fridley BL, Jenkins GD, Kalari KR, Lingle W, et al: FKBP51 affects cancer cell response to chemotherapy by negatively regulating Akt. Cancer Cell 2009, 16(3):259-266.

26. Eckerle S, Brune V, Doring C, Tiacci E, Bohle V, Sundstrom C, et al: Gene expression profiling of isolated tumour cells from anaplastic large cell lymphomas: insights into its cellular origin, pathogenesis and relation to Hodgkin lymphoma. Leukemia 2009, 23(11):2129-2138.

27. Chen DT, Nasir A, Culhane A, Venkataramu C, Fulp W, Rubio R, et al: Proliferative genes dominate malignancy-risk gene signature in histologically-normal breast tissue. Breast Cancer Res Treat 2010, 119(2):335-346.

28. Wurmbach E, Chen YB, Khitrov G, Zhang W, Roayaie S, Schwartz M, et al: Genome-wide molecular profiles of HCV-induced dysplasia and hepatocellular carcinoma. Hepatology 2007, 45(4):938-947.

29. Hou J, Aerts J, den Hamer B, van ljcken W, den Bakker M, Riegman P, et al: Gene expression-based classification of non-small cell lung carcinomas and survival prediction. PLOS One 2010, 5(4):e10312. 
30. Barrett T, Wilhite SE, Ledoux P, Evangelista C, Kim IF, Tomashevsky M, et al: $\mathrm{NCBI}$ GEO: archive for functional genomics data sets-update. Nucleic Acids Research 2013, 41(Database issue):D991-D995.

31. Hsu SD, Lin FM, Wu WY, Liang C, Huang WC, Chan WL, et al: miRTarBase: a database curates experimentally validated microRNA-target interactions. Nucleic Acids Research 2011, 39(Database issue):D163-D169.

32. Plaisier $\mathrm{CL}$, Pan $\mathrm{M}$, Baliga NS: A miRNA-regulatory network explains how dysregulated miRNAs perturb oncogenic processes across diverse cancers. Genome Research 2012, 22(11):2302-2314

33. Chang CC, Lin CJ: LIBSVM: A Library for Support Vector Machines. Acm T Intel Syst Tec 2011, 2(3)

34. Mi H, Muruganujan A, Casagrande JT, Thomas PD: Large-scale gene function analysis with the PANTHER classification system. Nature Protocols 2013, 8(8):1551-1566.

35. Shannon P, Markiel A, Ozier O, Baliga NS, Wang JT, Ramage D, et al: Cytoscape: a software environment for integrated models of biomolecular interaction networks. Genome Research 2003, 13(11):2498-2504.

doi:10.1186/1471-2164-16-S9-S4

Cite this article as: Kwon et al:: Integrative analysis of multi-omics data for identifying multi-markers for diagnosing pancreatic cancer. BMC Genomics 2015 16(Suppl 9):S4.

\section{Submit your next manuscript to BioMed Central} and take full advantage of:

- Convenient online submission

- Thorough peer review

- No space constraints or color figure charges

- Immediate publication on acceptance

- Inclusion in PubMed, CAS, Scopus and Google Scholar

- Research which is freely available for redistribution

Submit your manuscript at www.biomedcentral.com/submit 